\title{
Eye Part
}

National Cancer Institute

\section{Source}

National Cancer Institute. Eye Part. NCI Thesaurus. Code C13019.

Any component associated with the organ for sight or vision. 